\title{
Present Evidence for Intermediate Mass Black Holes in ULXs and Future Prospects
}

\author{
J. M. Miller \\ Harvard-Smithsonian CfA, 60 Garden Street, Cambridge MA 02138, USA; \\ jmmiller@cfa.harvard.edu
}

\begin{abstract}
In a number of the most luminous ULXs (those with $L_{X} \sim 10^{40} \mathrm{erg} \mathrm{s}^{-1}$ ) in nearby galaxies, observations with XMM-Newton and Chandra are revealing evidence which suggests that these ULXs may harbor intermediate-mass black holes (IMBHs). The detection of accretion disk spectral components with temperatures 5-10 times lower than the temperatures observed in stellar-mass black hole binaries near to their Eddington limit may be particularly compelling evidence for IMBH primaries, since $T \propto M^{-1 / 4}$ for disks around black holes. In some sources, X-ray timing diagnostics also hint at IMBHs. Evidence for IMBHs in a subset of the most luminous ULXs, a discussion of the robustness of this evidence and alternatives to the IMBH interpretation, and prospects for better determining the nature of these sources in the future, are presented in this work.
\end{abstract}

\section{Introduction to ULXs}

Ultraluminous X-ray sources (ULXs) are bright, off-nuclear point sources in nearby normal galaxies, for which the inferred X-ray luminosity exceeds the isotropic Eddington limit for a $10 M_{\odot}$ black hole $(L \simeq$ $1.3 \times 10^{39} \mathrm{erg} \mathrm{s}^{-1}$; Frank, King, \& Raine 2003). In most ULXs, longtimescale X-ray variability indicates that the ULXs are likely accreting black hole binaries like those known in the Milky Way. The existence of ULXs was first revealed with Einstein (Fabbiano 1989). Chandra and XMM-Newton have revolutionized the study of these sources by enabling observations which effectively isolate individual sources and which obtain sensitive spectra and lightcurves from the brightest nearby examples. For a recent review of ULXs, see, e.g., Fabbiano \& White (2005).

Among the reasons that ULXs are interesting is that they may represent rare phases of accretion in binary systems, rare X-rays states, and/or rare phases of binary evolution. However, the most compelling reason to study ULXs is likely because their luminosity suggests that they may harbor intermediate mass black holes (IMBHs, $10^{2-5} M_{\odot}$; for a recent review see Miller \& Colbert 2004). It is well-known that stellar-mass black holes can reach luminosities slightly in excess of their implied isotropic Eddington limit (see, e.g., McClintock \& Remillard 2005), so ULXs at the lower end of the luminosity range (indeed,

(c) 2018 Kluwer Academic Publishers. Printed in the Netherlands. 


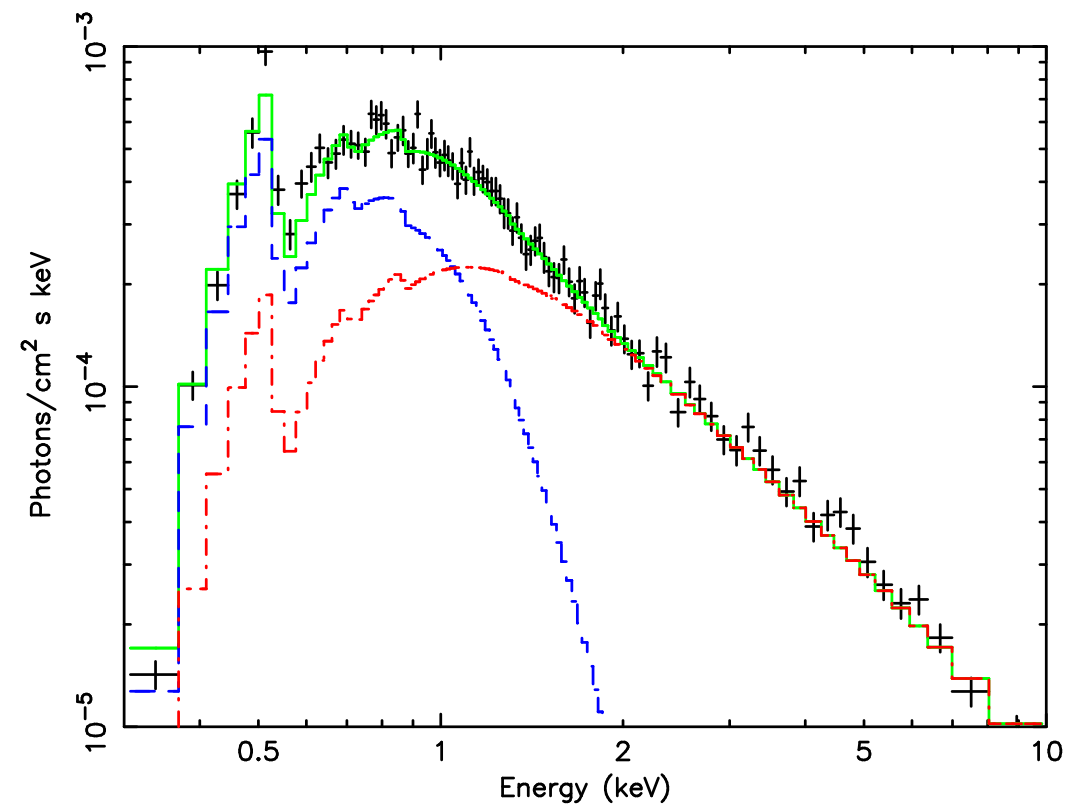

Figure 1. The XMM-Newton/EPIC-pn X-ray spectrum of NGC 1313 X-1 is shown above (Miller, Fabian, \& Miller 2004b). A disk component is shown in blue, and hard power-law emission is shown in red. In this source and a growing number of other ULXs, X-ray spectra require a cool disk component, which may be evidence for an accreting IMBH, e.g. in a binary system.

this is the majority of ULXs) are likely binaries with stellar-mass black hole primaries (or neutron star primaries in rare cases). However, those ULXs at the upper-end of the luminosity range (arbitrarily, $L_{X} \geq 10^{40} \mathrm{erg} \mathrm{s}^{-1}$ ) may harbor IMBHs. Recent observations with Chandra and XMM-Newton (in particular) have revealed cool accretion disk components in the spectra of the most luminous ULXs (see Miller, Fabian, \& Miller 2004a) and other X-ray spectral and timing features which suggest IMBHs. The status of the evidence for IMBHs in ULXs, arguments against IMBHs, and prospects for better understanding the $\mathrm{IMBH}$ candidates is discussed below.

\section{Evidence for IMBHs in ULXs}

Spectra of bright ULXs obtained with $A S C A$ were typically described in terms single flux components, and often in terms of hot accretion disk components (Makishima et al. 2000), although weak evidence for twocomponent spectra consisting of cool accretion disk and hard powerlaw flux components was also reported (Colbert \& Mushotzky 1999). Recent observations of the most luminous and proximal ULXs with 


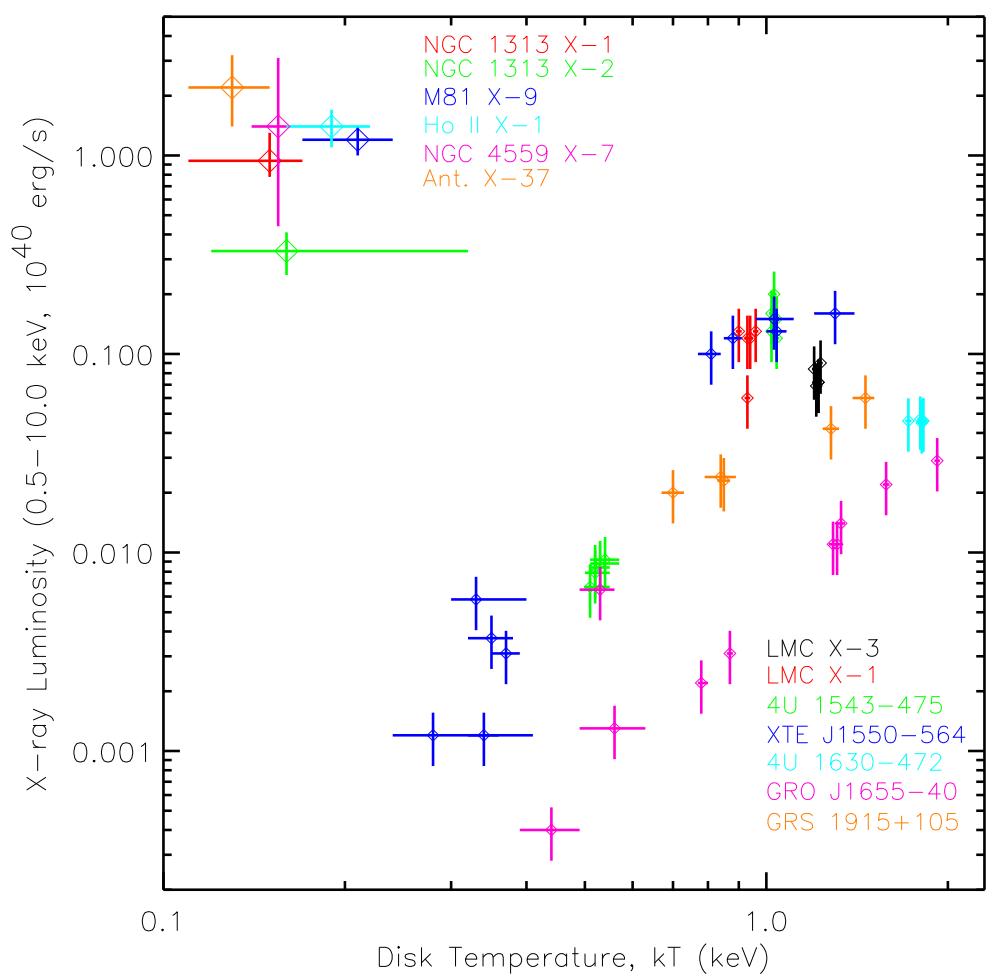

Figure 2. ULXs with cool accretion disks do not lie on the temperature-luminosity trend observed in stellar-mass black holes, and form a rather tight group, suggesting a distinct sub-class which may indeed harbor IMBHs (Miller, Fabian, \& Miller 2004a).

XMM-Newton and Chandra appear to have resolved this discrepancy: Miller et al. (2003) first reported the detection of a cool accretion disk at the $8 \sigma$ level of confidence in XMM-Newton spectra of NGC 1313 X1 (see Figure 1). Other significant detections of cool disk components have been reported in XMM-Newton and/or Chandra spectra of NGC 1313 X-2 (Miller et al. 2003), NGC 5408 X-1 (Kaaret et al. 2003), M81 X-9 (Holmberg IX X-1, Miller, Fabian, \& Miller 2004a), Antennae X37 (Miller et al. 2004), Holmberg II X-1 (Dewangan et al. 2004), NGC 4559 X-7 (Cropper et al. 2000), and most recently in a ULX in M101 (Kong, DiStefano, \& Yuan 2004).

In each of these cases, the luminosity of the ULX is near to or above $L_{X} \simeq 10^{40} \mathrm{erg} \mathrm{s}^{-1}$, and the measured disk color temperature is consistent with the $k T \simeq 0.1-0.2 \mathrm{keV}$ range. These ULX disk temperatures are much lower than the $k T \simeq 1-2 \mathrm{keV}$ disks temperatures commonly measured in $\sim 10 M_{\odot}$ black hole binaries in the 


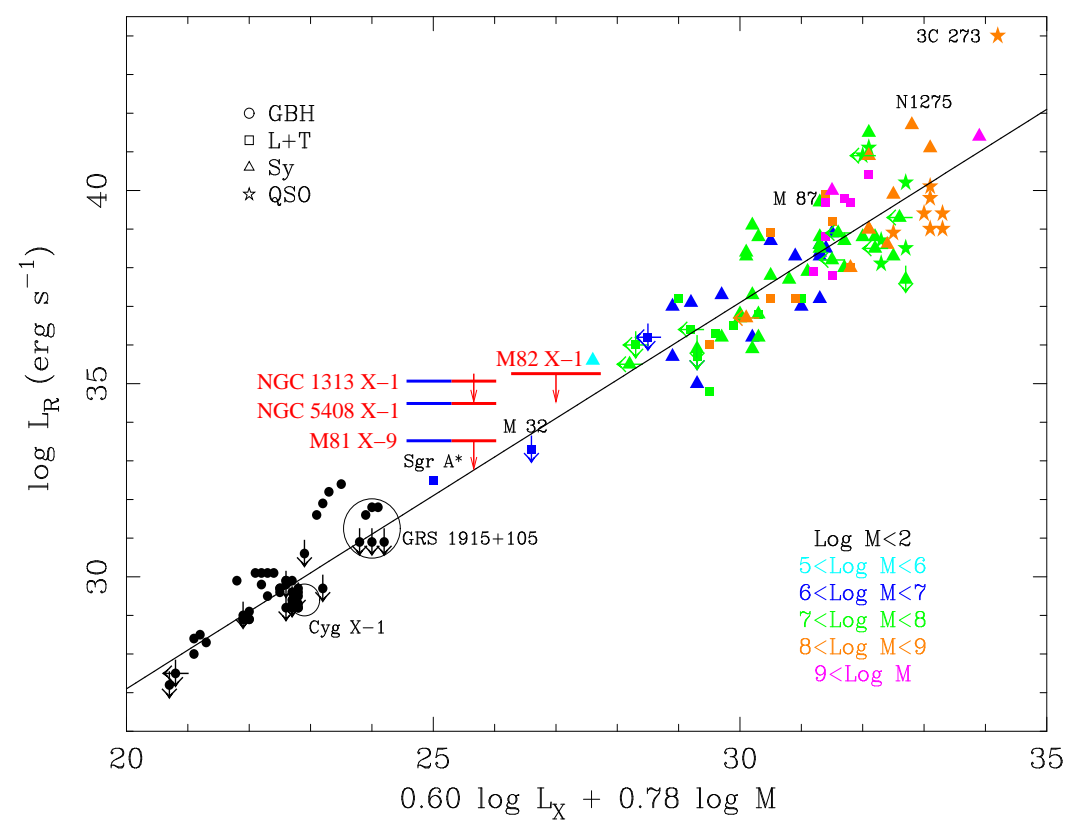

Figure 3. Merloni, Heinz, \& Di Matteo (2003) found a "fundamental plane" relating black hole mass, X-ray luminosity, and radio luminosity in unbeamed sources. Bright ULXs with cool disks and good limits on radio flux (NGC 1313 X-1: Colbert et al. 1995; M81 X-9: $F \leq 60 \mu J y$ at $5 \mathrm{GHz}$, Jimenez-Garate et al. 2005 in prep.) or radio detections (NGC 5408 X-1: Kaaret et al. 2003), and M82 X-1, fit between stellar-mass and supermassive black holes on the fundamental plane. For M82 X-1, the radio limits and lower mass bound are from Kaaret et al. (2001), and the upper mass bound is from Strohmayer \& Mushotzky (2003). For the other sources, red error bars represent a mass range of $300-3000 M_{\odot}$, a representative range for disk normalization and temperature scaling, respectively, and the blue error bars extend down to approximately the mass found from simple Eddington limit scaling. (The fundamental plane makes use of $2-10 \mathrm{keV}$ luminosity values and flux densities at 5 GHz. ULX luminosities reported in Miller, Fabian, \& Miller 2004a were converted to the $2-10 \mathrm{keV}$ band and flat radio spectra were assumed).

Milky Way and LMC when they are observed at luminosities near to their Eddington limit. Indeed, because $T \propto M^{-1 / 4}$ for standard disks around black holes (Frank, King, \& Raine 2002), the low disk temperatures measured in these ULXs imply black holes with masses in the $f e w \times 10^{2-3} M_{\odot}$ range when scaled to the temperatures seen in stellar-mass black hole binaries. (The normalizations of these disk components can also be scaled and generally imply IMBHs; see Miller et al. 2003.) These ULXs occupy a distinct region of a $L_{X}-k T$ diagram, which is distinct from the $L \propto T^{4}$ trend clearly seen in stellar-mass black holes (see Figure 2; see also Miller, Fabian, \& Miller 2004a). The exceptionally high luminosities inferred in these sources, their low disk 
temperatures, and their clustering in a $L_{X}-k T$ diagram makes these sources strong IMBH-candidates.

M82 X-1 may be the single best IMBH-candidate ULX presently known, in part due to its exceptionally high luminosity, which approaches $L_{X} \simeq 10^{41} \mathrm{erg} \mathrm{s}^{-1}$. However, the nature of its low energy spectrum is unknown due to contamination from a surrounding thermal plasma. Strohmayer \& Mushotzky (2003) have reported the detection of a $54 \mathrm{mHz}$ QPO in the X-ray flux of this ULX, based on observations with XMM-Newton and RXTE. As QPOs are thought to be disk oscillations, this feature indicates that the X-ray flux is not beamed, and implies the observed luminosity is a true isotropic luminosity, greatly reinforcing an IMBH interpretation.

A timing diagnostic has been interpreted as tentative evidence for an IMBH in at least one other case: Cropper et al. (2004) have reported a break frequency at $28 \mathrm{mHz}$ in the power density spectrum of NGC 4559 X-7. The break could indicate a mass of $38 M_{\odot}$ or $1300 M_{\odot}$, as there are two breaks commonly observed in the power spectra of accreting black holes (both in stellar-mass and supermassive black holes; see, e.g., Uttley, McHardy, \& Papadakis 2002). It is very difficult to obtain a mass scaling from either a single break or a single QPO, and indeed there is still some ambiguity when two breaks or multiple QPOs are detected since characteristic frequencies drift with flux.

\section{On the Robustness of Evidence for IMBHs in ULXs}

\subsection{Detalls of the Spectral Modeling}

Evidence for cool accretion disks in sources with luminosities near to $10^{40} \mathrm{erg} \mathrm{s}^{-1}$ is generally quite robust. The requirement for low disk temperature with normalizations also suggesting IMBH primaries does not depend the choice of disk model (Miller, Fabian, \& Miller 2004a). Cool disks are required regardless of whether independent flux components or self-consistent Compton up-scattering models are used to fit the X-ray spectra (Miller et al. 2003; Miller, Fabian, \& Miller 2004). An uncertainty in scaling from disks around stellar-mass black holes to disks around presumed IMBHs exists due to potential differences between the intrinsic disk spectrum and measured spectrum (after transfer through a disk atmosphere). However, it has recently been shown that these effects are remarkably similar in disks around stellarmass black holes and IMBHs (Fabian, Ross, \& Miller 2004). Finally, low metal abundances in absorbing material along the line of sight to these sources does not falsely create a statistical need for a cool disk 
component in the most sensitive spectra (Miller et al. 2003; Miller, Fabian, \& Miller 2004b).

As noted above, spectra of some of the most luminous ULXs obtained with $A S C A$ could be described only in terms of a single hot disk component (temperatures approached $2 \mathrm{keV}$; see Makishima et al. 2000). It is now clear that these sources are generally better described with a combination of a cool disk and hard power-law. Both Chandra and XMM-Newton have much narrower beams than $A S C A$, and so fold-in far less background (both diffuse and point-source in nature). Moreover, the lower energy bounds of Chandra and XMM-Newton are lower than the effective lower energy bound of $A S C A$, which gradually increased over the mission lifetime.

Stobbart, Roberts, \& Warwick (2004) recently fit the spectrum of a dipping ULX in NGC $55\left(L_{x}=1.6 \times 10^{39} \mathrm{erg} \mathrm{s}^{-1}\right)$ with a model consisting of a hot $(k T=0.8 \mathrm{keV})$ disk dominating the high energy spectrum, and a very soft $(\Gamma=4)$ power-law dominating the low energy spectrum. Some parameters were fixed in fits to the spectra of this ULX (this is not a standard practice), so it is not clear that a model consisting of a low temperature disk and hard power-law is inconsistent with the data (though the spectra of dipping sources can be confusing and non-standard). Aside from the fact that the spectral fitting method is non-standard, the model is inconsistent with the observed spectra of stellar-mass Galactic black hole binaries and Compton up-scattering models for hard X-ray emission (Stobbart, Roberts, \& Warwick note these facts).

Although this model is likely unphysical, it is interesting to test whether or not it might provide a reasonable alternative (in terms of a goodness-of-fit statistic) to spectra of IMBH-candidate ULXs which are presently well described in terms of cool disks and hard power-law components. Let us take NGC $1313 \mathrm{X}-1$ as an example. Jointly fitting the XMM-Newton pn, MOS1, and MOS2 spectra of NGC 1313 X-1 (see Miller et al. 2003; Miller, Fabian, \& Miller 2004) with a simple model consisting of multicolor disk black body and power-law components (modified by neutral absorption), the cool disk plus hard power-law model gives a very good fit: $\chi^{3} / d o f=885.8 / 870(k T=0.18 \mathrm{keV}$, $\Gamma=1.8$ ). Constraining the disk temperature to be $k T \geq 0.8 \mathrm{keV}$, the resultant fit is significantly worse statistically: $\chi^{2} / d o f=965.3 / 870$ $(k T=2.6 \mathrm{keV}, \Gamma=4.3)$.

As stated before, M82 X-1 is unlike other IMBH-candidate ULXs in that it is embedded in a region of strong diffuse plasma emission, which makes it very difficult to constrain the nature of its low energy spectrum (see Strohmayer \& Mushotzky 2003). Although other strong IMBH-candidate ULXs are not located within similar clusters of young 
stars, it is nevertheless important to understand the extent to which thermal plasmas may contribute to the low-temperature thermal emission. At low temperatures, an O VII or O VIII emission line should be particularly strong, and there is no evidence for such a line in the $\mathrm{X}$-ray spectra of IMBH-candidate ULXs (note that careful fitting of the $\mathrm{O}$ K-edge is required to prevent false O VII/VIII line detections; see Miller, Fabian, \& Miller 2004a). At present, no soft X-ray line has been reported in a ULX which is significant at or above the $3 \sigma$ level. Thus, it presently appears that the low-temperature thermal components detected in some ULXs are due to optically-thick disk components, but improved spectra are required to put strong constraints on possible contributions from an optically-thin thermal plasma.

\subsection{Relativistic Beaming}

Relativistic beaming of source flux has been proposed as a means of explaining luminosities in apparent excess of the isotropic Eddington limit for a $10 M_{\odot}$ black hole (Reynolds et al. 1997; Kording, Falcke, \& Markoff 2002). The very low radio to X-ray flux ratios found in luminous ULXs with cool disks strongly argues against this interpretation, since beaming tends to create flat $\nu \mathrm{F}_{\nu}$ spectra (e.g. in blazars, Miller et al. 2003; Kaaret et al. 2003; Miller, Fabian, \& Miller 2004). Indeed, the ratios found are below the maximum ratios observed in stellar mass black holes which are observed edge-on (see Fender \& Kuulkers 2001), rather than along a line of sight coincident with a jet axis. Blackbody components imply a minimum physical size, and here again the cool disks detected argue against relativistic beaming. Finally, it should be noted that in the special case of M82 X-1, the detection of QPO strongly argues that the disk is seen clearly, and therefore argues against relativistic beaming as a viable means of explaining the inferred luminosity (Strohmayer \& Mushotzky 2003).

It is interesting to consider how the broad-band properties of IMBHcandidate ULXs with constraining radio limits or detections compare to stellar-mass and supermassive black holes. Merloni, Heinz, \& DiMatteo (2003) examined the properties of a number of unbeamed black holes, and found a fundamental plane of black hole activity which relates mass, X-ray luminosity, and radio luminosity. Figure 3 shows the fundamental plane, with the addition of the IMBH-candidate ULXs NGC 1313 X-1, M81 X-9, NGC 5408 X-1, and M82 X-1. These black holes lie on the fundamental plane, or only slightly above the plane (but no farther than the scatter in the supermassive black holes population). This again implies that these sources are not beamed, and their position 
between the stellar-mass black hole population and supermassive black holes again suggests that they may indeed harbor IMBHs.

\subsection{Disk Issues: Funnels, Photon Bubbles, and Slim Disks}

It has been suggested that at very high mass accretion rates, a funnel may form at the inner disk and boost luminosities by factors of 10-30 along the funnel axis (King et al. 2001). This model would allow $10 M_{\odot}$ black holes to apparently violate the isotropic Eddington limit, and could explain the high apparent luminosities seen in some ULXs. The low disk temperatures measured in some IMBH-candidate ULXs already argue against this model, because disk temperatures in the 0.1$0.2 \mathrm{keV}$ range would only signal a high mass accretion rate for IMBHs. Observations of stellar-mass galactic black holes also argue against the formation of such structures: $4 \mathrm{U} 1543-475$ is a Galactic black hole binary viewed at $i=21^{\circ}$, but it does not exceed its Eddington limit by more than a factor of a few (Miller, Fabian, \& Miller 2004b; Park et al. 2004). Stronger funneling might be required, but radiation will leak out the sides of a strong funnel geometry as it becomes ionized and optically-thin, ruining the funnel effect (King \& Pounds 2003). Finally, in the case of Holmberg II X-1, optical spectroscopy of a surrounding nebula reveals that the isotropic luminosity must exceed the Eddington limit for a $10 M_{\odot}$ black hole (Kaaret, Zezas, \& Ward 2004), and the morphology of optical nebulae around other ULXs does not strongly suggest beaming.

It has also been suggested that radiation pressure-dominated disks might be able to produce super-Eddington fluxes through small-scale photon bubble instabilities (Begelman 2002). Slim disk solutions (e.g. Watarai, Mizuno, \& Mineshige 2001) may also allow high fluxes. Where IMBH-candidate ULXs are concerned, the difficulty with both models is that they are expected to hold at very high mass accretion rates when disks are expected to be hot. The low disk temperatures observed in some IMBH-candidate ULXs means that photon-bubble disk and/or slim disks are not required in these sources.

\subsection{Photospheres}

It has recently been suggested that the basic disk plus corona geometry inferred in black hole systems accreting at high rates may be incorrect, and that a better model may consist of an outflowing photosphere which is optically thick at $r \leq 100 R_{S c h w}$. and external shocks generating hard X-rays (King \& Pounds 2003). In any case where the flux in the hard spectral component is equal to the flux in the soft component, the photosphere would have to flow at $v=c$ and the photospheric radius 
would have to equal $1 R_{S c h w}$. to generate the observed hard X-ray flux, which renders this alternative implausible in most ULXs with cool disks and luminous quasars. Weak X-ray absorption lines in the spectra of some AGN - apparently blue-shifted in the frame of the AGN and cited as evidence in favor of outflowing photospheres - have recently been shown to coincide with the AGN recession velocity in a number of cases, demanding absorption near to the Milky Way (McKernan, Yaqoob, \& Reynolds 2004). Thus, both in the case of AGN and ULXs, the data would seem to argue against this alternative in the majority of cases.

It should be noted that sources like the bright, soft transient in M101 (Kong, DiStefano, \& Yuan 2004), as well as "super-soft" sources and "qausi-soft" sources, do not have significant hard components and the photosphere model cannot be excluded in these cases. Apart from the immediate issue of photospheres, the very low flux observed from super-soft and quasi-soft sources makes it nearly impossible to obtain strong spectral and timing constraints, and inferences for IMBHs in these sources require extreme caution as they are necessarily at the level of what the data will allow, rather than what the data strictly require.

\subsection{InFERENCES FROM X-RAY LUMinOSITY FunCtions}

It is sometimes argued that X-ray luminosity functions demonstrate that all ULXs are stellar-mass X-ray binaries, because the highest luminosity sources appear to extend naturally from the lower-luminosity distribution without a break (see, e.g., Swartz et al. 2004). Several assumptions are implicit in such an argument: first, that IMBHs should have a narrow mass range (a wide range of masses would act to diminish a break); second, that IMBHs should all be accreting at high fractions of their Eddington limit (a range of mass accretion rates like that seen in stellar-mass binaries in the Milky Way and Magellanic Clouds would also act to flatten a break); and third, that a single observation or a few observations can reliably constrain the nature of a source population that is likely to be variable.

The high luminosity end of the X-ray luminosity function of a given galaxy is a regime with very few sources, where strong constraints are not possible. The statistics do not allow one to strongly require or to exclude a break. Consider how different the X-ray luminosity functions of galaxies like M101 (Kong, DiStefano, \& Yuan 2004) and NGC 3628 (Strickland et al. 2001) must appear when their transient ULXs (which reach near to or above $L_{X} \simeq 10^{40} \mathrm{erg} \mathrm{s}^{-1}$ ) vary by factors of 10 and 1000, respectively. Again, as this regime is a low-statistics regime, it is unlikely that one source or even two sources could require a break, but it 
illustrates the danger of drawing conclusions about source populations based on X-ray luminosity functions. In fact, the statistical uncertainty of X-ray luminosity functions is worse than typically presented: errors on luminosity resulting from uncertainties in the absorbing column and differences between spectral models that cannot be distinguished in poor quality spectra are not generally considered.

X-ray luminosity functions do indicate that only a small number of IMBHs are accreting at high fractions of their Eddington limit at any given time in a typical galaxy. This inference is broadly consistent with the behaviors observed from stellar mass and supermassive black holes. Moreover, X-ray luminosity functions may demonstrate that the population of IMBHs which may exist in binaries is likely small relative to the number of black holes and neutron stars in accreting binaries. These inferences are limited, but as such they are fair to the limited statistics at the high luminosity end of X-ray luminosity functions.

\section{Future Prospects}

It will likely prove to be very difficult to obtain optical/IR radial velocity curves of ULXs to constrain the mass of the primary. Putting even the brightest stellar-mass Galactic binaries at distances of a few Mpc makes them very faint indeed. Further complications arise because most IMBH-candidate ULXs - in contrast to most stellar-mass Galactic black holes - are persistently active; light from the accretion disk will make it difficult to identify and trace features from the companion star. If ULXs are very wide binaries, the long orbital period will make it even harder to obtain radial velocity curves. The optical nebulae found around some IMBH-candidate ULXs (Pakull \& Mirioni 2003) may further complicate spectroscopic studies of the presumed binary system. For the foreseeable future, it is likely that the nature of IMBHcandidate ULXs will be decided based on a preponderance of indirect evidence.

From an X-ray point of view, much longer observations of galaxies harboring IMBH-candidate ULXs are urgently needed. Observations of $300-500 \mathrm{ksec}$ (and longer) have been devoted to the study of relativistic effects in accreting sources (e.g. $500 \mathrm{ksec}$ to study putative absorption

lines from the surface of the neutron star in EXO 0748-676, nearly 400 ksec to study the broad Fe K $\alpha$ emission line in MCG-6-30-15, and nearly 300 ksec to study the broad Fe K $\alpha$ emission line in GX 339-4). Establishing the presence or absence of IMBHs - a new class of relativistic objects - is a goal as deserving of long observations as the study of phenomena in known classes of relativistic objects. 
For ULXs within a few Mpc with $L_{X} \geq 10^{40} \mathrm{erg} \mathrm{s}^{-1}$, an XMMNewton observation of 300-500 ksec will achieve the sensitivity required to: (1) detect a broad $\mathrm{Fe} \mathrm{K} \alpha$ emission line from the accretion disk, which would provide independent evidence for a standard accretion disk and rule-out relativistic and geometric beaming; (2) detect breaks in the power-density spectrum and/or QPOs, which will enable independent mass estimates based on scaling the characteristic frequencies (indeed, the relation between QPO frequency and break frequency in Galactic black holes and neutron stars found by Wijnands \& van der Klis 1999 may provide an additional pragmatic scaling beyond scalings based only on breaks); and (3) detect any soft X-ray emission lines, enabling the (likely small) flux of any diffuse optically-thin plasma to be separated from an underlying optically-thick disk continuum.

Of course, far better radio and optical constraints are also required. Even though optical/IR radial velocity curves may be difficult to obtain, the value of spectroscopic studies of the nebulae surrounding some ULXs is clear (see Pakull \& Mirioni 2003; Kaaret, Ward, \& Zezas 2004). Independent luminosity and beaming constraints can impact our view of the nature of IMBH-candidate ULXs. Better radio constraints are needed to rule-out beaming in more sources. Radio detections may not be possible in some cases, but strong limits allow sources to be placed on the "fundamental plane" (for instance), and the position of sources on the "fundamental plane" does reflect their nature (though it does not allow for a precise mass measurement). Mushotzky (2004) and collaborators have undertaken a radio survey of ULXs, and in some cases have found broad contours coincident with the X-ray source positions; the lack of strongly peaked point source emission in most cases may again argue against beaming.

$\mathrm{X}$-rays probe the regions closest to compact objects, and can be expected to have the greatest impact on our understanding of ULXs and IMBH-candidate ULXs in particular. In the long run, planned missions like Constellation- $X$ and $X E U S$ will revolutionize the study of ULXs. These missions will make it possible to obtain sensitive spectroscopic and timing constraints on ULXs with short observations. Relativistic reverberation mapping in AGN may be a primary goal of missions like Constellation- $X$ and $X E U S$, but for a variety of possible designs, these missions will be able to reveal or reject the IMBH hypothesis in a much higher number of ULXs. 


\section{Summary}

The notorious M82 X-1 and at least six ULXs with luminosities near to or above $L_{X} \simeq 10^{40} \mathrm{erg} \mathrm{s}^{-1}$ and soft components consistent with optically-thick emission from cool $(k T=0.1-0.2 \mathrm{keV})$ accretion disks (Miller, Fabian, \& Miller 2004b) may be regarded as IMBH-candidates. The properties which have been observed to separate these ULXs from populations of stellar-mass and supermassive black holes appear to be robust. In the cases where they can be strongly tested, alternative explanations for the high inferred luminosities and the nature of the spectra appear to be implausible. This proceedings has attempted to take a critical view of the evidence supporting an IMBH explanation for a subset of very luminous ULXs, but also a critical view of the counter-arguments. Dramatic strides towards resolving the nature of these sources can be made in the near future with optical and radio observations, and principally with very long X-ray observations.

\section{Acknowledgements}

Thanks to A. C. Fabian and M. C. Miller for collaboration and discussions. Thanks to A. Merloni for his generous permission to use

the fundamental plane figure, and also to M. Jimenez-Garate for his generous communication of the radio limit on M81 X-9. Thanks to P. Martinez. Final thanks to the National Science Foundation for support through their Astronomy \& Astrophysics Postdoctoral Fellowship.

\section{References}

Begelman, M., 2002, ApJ, 538, L97

Colbert, E. J. M., \& Mushotzky, R. F., 1999, ApJ, 519, 89

Cropper, M. C., Soria, R., Mushotzky, R., Wu, K., Markwardt, C., \& Pakull, M., 2004, MNRAS, 349, 39

Dewangan, G., Miyaji, T., Griffiths, R. E., \& Lehmann, I., 2004, ApJ, 608, L57

Fabian, A. C., Ross, R. R., \& Miller, J. M., 2004, MNRAS, 335, 359

Fabbiano, G., 1989, ARA\& A, 27, 87

Fabbiano, G., \& White, N. E., 2005, to appear in "Compact Stellar X-ray Sources" eds. M. van der Klis and W. H. G. Lewin, Cambridge Univ. Press, astro-ph/0307077

Fender, R. P., \& Kuulkers, E., 2001, MNRAS, 324, 923

Frank, J., King, A. R., \& Raine, D., 2002, in "Accretion Power in Astrophysics", Cambridge: Cambridge Univ. Press Kaaret, P., et al., 2001, MNRAS, 321, L29

Kaaret, P., Corbel, S., Prestwich, A. H., \& Zezas, A., 2003, Science, 299, 365

Kaaret, P., Ward, M. J., \& Zezas, A., 2004, MNRAS, 351, 83

King, A. R., Davies, M. B., Ward, M. J., Fabbiano, G., \& Elvis, M., 2001, ApJ, 
552, L109

King, A. R., \& Pounds, K., 2003, MNRAS, 345, 657

Kording, E., Falcke, H., \& Markoff, S., 2002, A\& A, 382, L13

Kong, A. K. H., DiStefano, R., \& Yuan, F., 2004, ApJ, 617, L49

Makishima, K., et al., 2000, ApJ, 535, 632

McClintock, J. E., \& Remillard,a R. A., 2005, to appear in "Compact Stellar X-ray Sources" eds. M. van der Klis and W. H. G. Lewin, Cambridge Univ. Press, astro-ph/0306213

McKernan, B., Yaqoob, T., \& Reynolds, C. S., 2004, ApJ, subm., astro-ph/0408506

Miller, J. M., Fabbiano, G., Miller, M. C., \& Fabian, A. C., 2003, ApJ, 585, L37

Miller, J. M., Fabian, A. C., \& Miller, M. C., 2004a, ApJ, 614, L117

Miller, J. M., Fabian, A. C., \& Miller, M. C., 2004b, ApJ, 607, 931

Miller, J. M., Zezas, A., Fabbiano, G., \& Schweizer, F., 2004, ApJ, 609, 782

Miller, M. C., \& Colbert, E. J. M., 2004, IJMPD, 2004, 1

Mushotzky, R., 2004, to appear in the proceedings of Kyoto, 2003, astro-ph/0411040

Pakull, M., \& Mirioni, L., 2003, in "New Visions of the X-ray Universe in the XMM-Newton and Chandra ERA" (ESA SP-488; Nordwijk: ESA), astro-ph/0202488

Park S. Q., et al., 2004, ApJ, 610, 378

Reynolds, C. S., Loan, A. J., Fabian, A. C., Makishima, K., Brandt, W. N., \& Mizuno, T., 1997, MNRAS, 286, 349

Stobbart, A.-M., Roberts, T. P., \& Warwick, R. S., 2004, MNRAS, 351, 1063

Strickland, D., Colbert, E. J. M., Heckman, T. M., Weaver, K. A., Swartz, D. A., Ghosh, K. K., Tennant, A. F., \& Wu, K., 2004, ApJS, 154, 519

Dahlem, M., \& Stevens, I. R., 2001, ApJ, 560, 707

Uttley, P., McHardy, I. M., \& Papadakis, I. E., 2002, MNRAS, 332, 231

Watarai, K., Mizuno, T., \& Mineshige, S., 2001, ApJ, 549, L77 
miller_edited.tex; 6/11/2018; 7:15; p.14 